UFIFT-HEP-96-9

hep-ph/9603272

March 1996

\title{
New Dark Matter Candidates Motivated From Superstring Derived Unification
}

\author{
Sanghyeon Changđ, Claudio Corianòn and Alon E. Faraggi谓 \\ Institute For Fundamental Theory, \\ Department of Physics, \\ University of Florida, \\ Gainesville, FL 32611 USA
}

\begin{abstract}
Perturbative gauge coupling unification in realistic superstring models suggests the existence of additional heavy down-type quarks, beyond the minimal supersymmetric standard model. The mass scale of the heavy down-type quarks is constrained by requiring agreement between the measured low energy gauge parameters and the string-scale gauge coupling unification. These additional quarks arise and may be stable due to the gauge symmetry breaking by "Wilson lines" in the superstring models. We argue that there is a window in the parameter space within which this down-type quark is a good candidate for the dark matter.
\end{abstract}

\footnotetext{
* E-mail address: schang@phys.ufl.edu

$\dagger$ E-mail address: coriano@phys.ufl.edu

‡ E-mail address: faraggi@phys.ufl.edu
} 
Substantial observational evidence indicates that most of the mass in the universe is invisible. The determination of the nature of this "dark matter" is one of the most important challenges confronting modern physics. In this paper we study the possibility that the dark matter is composed of heavy QCD color triplets that arise in standard-like superstring models. These are regular down-like heavy quarks with the down-type charge assignment. The existence of such heavy quarks is motivated from string-scale gauge coupling unification [1]. Due to its role in the string unification, we refer to this type of particle as the uniton. The uniton can be stable due to the breaking of gauge symmetries by "Wilson lines" in superstring models. The additional down-like quarks are obtained in the superstring models from sectors that arise due to the "Wilson line" breaking. As a result they acquire "fractional" charges under the $U(1)_{Z^{\prime}}$ gauge symmetry while the Standard Model states have the standard $S O(10)$ charge assignment. The stability of the uniton results from a gauge $U(1)$ symmetry, which is left unbroken down to low energies, or from a local discrete symmetry [2]. It forms bound heavy meson states with the Standard Model down and up quarks. We estimate the electromagnetic mass difference between the charged and neutral heavy $\mathrm{U}$-meson states and argue that, over some region of the parameter space, the neutral meson state is the lighter one. We estimate the contribution of the uniton to the relic density. We examine other astrophysical and terrestrial bounds on a stable uniton, and propose that there exist a window in the parameter space in which the uniton is a good dark matter candidate. In this paper we shall present our main results and further details of the analysis will be given in ref. [3].

In their low energy limit, heterotic string theories give rise to $N=1$ supersymmetry. While other possible extensions of the Standard Model are highly constrained or ruled out by experiments, supersymmetric theories are in agreement with the available data. The attractive motivation for supersymmetric theories is not without flaw. While the unification of the gauge coupling in the Minimal Supersymmetric Standard Model (MSSM) occurs at a scale $M_{\text {MSSM }} \approx 2 \times 10^{16} \mathrm{GeV}$, string theories predict a larger unification scale [4], typically $M_{\text {string }} \approx g_{\text {string }} \times 5 \times 10^{17} \mathrm{GeV}$ where $g_{\text {string }} \approx 0.8$ at the unification scale. Thus, an order of magnitude separates the MSSM and string 
unification scales.

It would seem that in an extrapolation of the gauge parameters over fifteen orders of magnitude, a problem involving a single order of magnitude would have many possible resolutions. Indeed, in superstring models there are a priori many possible effects that can account for the discrepancy. Surprisingly, however, the discrepancy is not easily resolved. The validity of string gauge coupling unification must be examined in the context of realistic string models. The superstring models in the free fermionic formulation represent a class of phenomenologically appealing models. Not only do these models naturally yield three generation with a plausible fermion mass spectrum, but perhaps of equal importance is the fact that these models predict $\sin ^{2} \theta_{W}=3 / 8$ at the string unification scale. This rather common result from the point of view of regular GUT models is highly non trivial from the point of view of string models. In ref. [1] it was shown, in a wide range of realistic free fermionic models, that heavy string threshold corrections, non-standard hypercharge normalizations, light SUSY thresholds or intermediate gauge structure, do not resolve the problem. Instead, the problem may only be resolved due to the existence of additional intermediate matter thresholds, beyond the MSSM [5, 6]. This additional matter takes the form of color triplets and electroweak doublets, in vector-like representations. Remarkably, some string models contain in their massless spectrum the additional states with the specific weak hypercharge assignments, needed to achieve string scale unification [6]. Possible scenarios to generate the needed mass scales from the string models have been discussed in the literature.

A model in the free fermionic formulation is generated by a consistent set of boundary condition basis vectors [7]. The physical spectrum is obtained by applying the generalised GSO projections. The first five basis vectors in our models consist of the so-called "NAHE-set" $\left\{\mathbf{1}, S, b_{1}, b_{2}, b_{3}\right\}$ 8]. At the level of the NAHE set the gauge group is $S O(10) \times S O(6)^{3} \times E_{8}$, with 48 generations. The number of generations is reduced to three and the $S O(10)$ gauge group is broken to $S U(3) \times S U(2) \times U(1)^{2}$ by adding to the NAHE set three additional basis vectors, $\{\alpha, \beta, \gamma\}$. The basis vectors $\alpha$ and $\beta$ break the $S O(10)$ symmetry to $S O(6) \times S O(4)$. The basis vector $\gamma$ breaks the 
$S O(2 n)$ symmetries to $S U(n) \times U(1)$. It is useful to note the correspondence between free fermionic models and orbifold models. The free fermionic models correspond to toroidal $Z_{2} \times Z_{2}$ orbifold models with nontrivial background fields. The NeveuSchwarz sector corresponds to the untwisted sector, and the sectors $b_{1}, b_{2}$ and $b_{3}$ correspond to the three twisted sectors of the orbifold models. The three sectors which break the $S O(10)$ symmetry correspond to Wilson lines in the orbifold terminology.

The massless spectrum of the superstring standard-like models consists of three 16 representations of $S O(10)$ from the sectors $b_{1}, b_{2}$ and $b_{3}$ decomposed under the final gauge group. The Neveu-Schwarz sector produces three pairs of electroweak doublets and several $S O(10)$ singlet fields. The sector $b_{1}+b_{2}+\alpha+\beta$ produces one or two additional electroweak doublet pairs and $S O(10)$ singlet fields. Additional massless states are obtained from sectors that arise from combinations of the vectors $\{\alpha, \beta, \gamma\}$ with the vectors of the NAHE set. The sectors $b_{j}+2 \gamma(j=1,2,3)$ produce three 16 representations of the hidden $S O(16)$ gauge subgroup decomposed under the final hidden gauge group. All the states above can either fit into $S O(10)$ multiplets or are $S O(10)$ singlets.

The massless spectrum of the superstring models contain additional massless states that do not fit into $S O(10)$ multiplets. For example, in the model of ref. [6] the sector $1+\alpha+2 \gamma$ produces a pair of color triplets with quantum numbers $(3,1,1 / 6)$ and $(\overline{3}, 1,-1 / 6)$ under $S U(3) \times S U(2) \times U(1)_{Y}$. The sectors $1+b_{1,2,3}+\alpha+2 \gamma$ produce three pairs of electroweak doublets with charges $(1,2,0)$. Such states are obtained from sectors that break the $S O(10)$ symmetry to $S O(6) \times S O(4)$ and therefore also appear in the $S O(6) \times S O(4)$ superstring models [9]. These states carry fractional electric charge and cannot be candidates for dark matter, as there are strong constraints on their possible mass scale and abundance.

In addition to the states above there is an additional class of massless states that are more interesting from cosmological considerations. These states are regular downlike quarks with the down-type charge assignments. These states are obtained from sectors that break the $S O(10)$ symmetry to $S U(3) \times S U(2) \times U(1)^{2}$. For example, in the model of ref. [6] the sectors $b_{1}+b_{2,3}+\beta \pm \gamma$ produce two pairs of color triplets 
with charges $(3,1,1 / 3)$ and $(\overline{3}, 1,-1 / 3)$. These states arise in the string models from sectors that break the $S O(10)$ symmetry. They carry fractional charges under the $U(1)_{Z^{\prime}}$ symmetry, which is embedded in $S O(10)$ and is orthogonal to $U(1)_{Y}$. Consequently, these color triplets cannot fit into $S O(10)$ multiplets. This property enables the stability of this type of color triplets. To examine whether these states can decay into the Standard Model states we must examine their interactions. For example, examination of the superpotential terms of the model of ref. [10] shows that such interactions terms are obtained if the $U(1)_{Z^{\prime}}$ symmetry is broken. The near stability of the uniton in this case can be associated with the existence of a low energy $Z^{\prime}$ gauge boson. However, this need not be the case. Analysis of the nonrenormalizable terms in the model of ref. [6] shows that superpotential terms between the uniton and the standard model states are not generated at any order [11]. In this model the uniton cannot decay into the standard model states even if the $U(1)_{Z^{\prime}}$ symmetry is broken. Therefore, in this model the uniton is stable. In this model the stability of the uniton may be associated with a local discrete symmetry [2].

The uniton forms bound meson states with the up and down quarks. In order to estimate the mass splitting of these two heavy mesons, we borrow from recent work of ref. [12 which gives a general formula for the mass splitting of two heavy-light mesons $(Q \bar{u}, Q \bar{d})$. We set $\Delta M=M^{-}-M^{0}$ to be the mass difference between the negatively charged meson and the zero charged one respectively. Intuitively, there are 2 contributions of opposite sign which contribute to the splitting : the difference in mass between the two constituent quarks, and the electromagnetic splitting. The basic strategy [12] is to relate the order $e^{2}$ isospin breaking corrections to the forward Compton scattering amplitude, $T$. The heavy quark effective theory, in the large $N_{c}$ limit, is then used to write $T$ in terms of heavy meson form factors by introducing a dispersion relation. The largest contribution to the spectral function comes from the lowest-lying single heavy meson states, while the continuum contribution is negligible. The infinite sum over the intermediate states is truncated after the first few terms, and includes, beside the contribution of the first excitation of the heavy meson, also 
a minimal sets of light mesons $\rho, \rho^{\prime} \omega$ and $\omega^{\prime}$. These are introduced in order to obtain consistency (at large $N_{c}$ ) with the asymptotic behaviour of the form factors from the heavy quark theory, as expected from the dimensional counting rules.

In the case of our heavy meson system the $\mathcal{O}\left(1 / m_{Q}^{2}\right)$ corrections are negligible. The electromagnetic contribution to the mass difference, up to $30 \%$ accuracy, is [12

$$
\begin{aligned}
M_{U^{+}}-\left.M_{U^{0}}\right|_{E M} \sim & +1.7 \\
-0.13 & \left(\frac{\beta}{1 \mathrm{GeV}^{-1}}\right)-0.03\left(\frac{\beta}{1 \mathrm{GeV}^{-1}}\right)^{2},
\end{aligned}
$$

where $\beta \sim 1 / m_{Q}$ measures the matrix element of the decay of the first excited heavy meson state into the ground state plus a photon. Using the values from the particle data book for $m_{u}, m_{d}$ and $m_{u} / m_{d}$ [13] we obtain $M_{U^{+}}-M_{U^{0}} \sim 1 \mathrm{MeV}$. The result given in eq. (11) does not include the contribution to the mass difference coming from $S U(2)$ splitting of the two constituent quarks inside the two mesons nor the strong interactions effects. An exact calculation of this splitting is not possible with our present understanding of low energy QCD. It is not unplausible that the electromagnetic mass difference and the strong interaction effects are of the order of a few $\mathrm{MeV}$ and can overcompensate the mass difference due to the two constituent quarks in the two mesons, therefore making $U^{+}$heavier than $U^{0}$. It is plausible that the splitting between $U^{+}$and $U^{0}$ behaves similarly to the B-meson case, in which the mass difference between $B^{+}$and $B^{0}$ is comparable to zero at the one sigma confidence level. We refer to [3] for a more detailed discussion of this issue. We therefore conclude that the possibility of having a neutral heavy meson lighter than the charged one is not ruled out.

We now discuss the cosmological and astrophysical bounds. The uniton is a strongly interacting particle and therefore it remains in thermal equilibrium until it becomes nonrelativistic. To calculate the relic density of the uniton we need to know its decoupling temperature from the thermal bath. In the nonrelativistic limit, $T / M<1$, the uniton annihilation rate is given by

$$
\Gamma=<\sigma|v|>n_{e q} \simeq \frac{\pi N \alpha_{s}^{2}}{M^{2}} n_{e q}
$$


where $M$ is the mass of the uniton, $\alpha_{s}$ is the strong coupling at decoupling, $n_{e q}$ is the number density of the uniton at equilibrium. $N$ is a summation over all the available annihilation channels and is given by $N=\sum_{f} a_{f}$. The amplitudes $a_{f}$ are obtained by calculating the annihilation cross section of the uniton to all the strongly interacting particles, which include the six flavors of quarks and squarks and the gluons and the gluinos. The final states are taken to be massless. We obtain $a=4 / 3$ for quarks; $a=14 / 27$ for gluons; $a=2 / 3$ for squarks and $a=64 / 27$ for gluinos.

The uniton decouples from the thermal bath when its annihilation rate falls behind the expansion rate of the universe. In the expanding universe, the evolution equation of the particle density in comoving volume is

$$
\frac{d Y}{d x}=-\lambda x^{-2}\left(Y^{2}-Y_{e q}^{2}\right)
$$

Here $Y=n / s, x \equiv M / T$ and

$$
\lambda=\left.\frac{x<\sigma|v|>s}{H}\right|_{x=1}=0.83 N \alpha_{s}^{2} \frac{g_{*} s}{\sqrt{g_{*}}} \frac{m_{p l}}{M} .
$$

Here the entropy $s$ is $\left(2 \pi^{2} / 45\right) g_{* s} m^{3} x^{-3}$. The decoupling condition $d Y / d x \simeq 0$ gives 14

$$
x_{d e c}=\ln [(2+c) \lambda a c]-\frac{1}{2} \ln \{\ln [(2+c) \lambda a c]\},
$$

where $a=0.145\left(\mathrm{~g} / g_{* s}\right)$ and $c$ is $Y\left(T_{d e c}\right) / Y_{e q}\left(T_{d e c}\right)$, which is of order one. We approximately estimate the decoupling temperature to be of the form

$$
T_{d e c} \simeq \frac{M}{\ln \left(m_{p l} / M\right)} .
$$

The uniton density at the present universe is

$$
Y_{0}=\frac{3.79 x_{d e c}}{\sqrt{g_{*}} m_{p l} M<\sigma|v|>},
$$

where we set $g_{*}=g_{* s}$, since the decoupling temperature is high. Since the relic energy density of a massive decoupled particle is $\rho_{0}=M s_{0} Y_{0}$, we can estimate the ratio of energy density to the critical energy density at the present universe to be

$$
\Omega_{0} h^{2} \equiv \frac{\rho h^{2}}{\rho_{c}} \simeq 10^{9} \frac{\ln \left(m_{p l} / M\right) M^{2}}{N \alpha_{s}^{2} \sqrt{g_{*}} m_{p l}} \mathrm{GeV}^{-1} .
$$


The cosmological data indicates that $0.1<\Omega h^{2}<1$. Using this condition we get an upper bound on the mass of the uniton

$$
M<10^{5} \alpha_{s}\left(N \sqrt{g_{*}} \ln \left(m_{p l} / M\right)\right)^{1 / 2} \mathrm{GeV}
$$

We now discuss the case with inflation and the decoupling temperature is greater than the reheating temperature. If the reheating temperature $T_{R}$ is smaller than the decoupling temperature $T_{d e c}$, those particles will be diluted away and regenerated after reheating by out-of-equilibrium production. Since the uniton is completely diluted after the inflation, the relic density at the reheating temperature is 0 . We can approximate it as

$$
\frac{d Y}{d x}=\lambda x^{-2} Y_{e q}^{2}
$$

with $Y_{e q}=0.145 \mathrm{~g} / g_{*} x^{3 / 2} e^{-x}$. Integrating this relation from the reheating temperature to the present temperature we get

$$
Y_{0}=\frac{\lambda g^{2}}{2}\left(\frac{0.145}{g_{*}}\right)^{2}\left(x_{r}+\frac{1}{2}\right) e^{-2 x_{r}},
$$

where $x_{r} \equiv M / T_{R}$. $T_{R}$ is the reheating temperature, and

$$
\Omega_{0} h^{2} \simeq 9 \times 10^{3} N \alpha_{s}^{2} g^{2} \frac{m_{p l}}{M}\left(\frac{200}{g_{*}}\right)^{1.5}\left(x_{r}+\frac{1}{2}\right) e^{-2 x_{r}} .
$$

We can estimate the bound on the mass,

$$
M>T_{R}\left[25+\ln \left(\sqrt{M / T_{R}}\right)\right]
$$

Without inflation, we have a strict bound on the mass of the uniton, which is around $10^{5} \mathrm{GeV}$. Inflation can raise the mass bound to any arbitrary order, depending upon the estimated value of the reheating temperature.

We remark that there are 3 windows $W 1, W 2$ and $W 3$ for strongly interacting dark matter [15] which possibly meet our requirements. In W1 we need $10 \mathrm{GeV}$ $<M<10^{4} \mathrm{GeV}$ and a scattering cross section of the heavy hadron to proton about $10^{-24} \sim 10^{-20} \mathrm{~cm}^{2}$. In W2 and W3 we need $10^{5} \mathrm{GeV}<M<10^{7} \mathrm{GeV}$ and $M>10^{10}$ $\mathrm{GeV}$, respectively, assuming a cross section less than $10^{-25} \mathrm{~cm}^{2}$. A charged bound 
state will form a hydrogen-like atom and have a cross section about $\sim 10^{-16} \mathrm{~cm}^{-2}$. The upper bound on the cross section is fixed by the neutron star lifetime [16]. Note that there are no limits from nucleosynthesis on a nonrelativistic heavy uniton because of its low density relative to proton and neutron and its low energy density compared with the energy density of radiation at nucleosynthesis. COBE data fits well with $\Omega_{0} h \simeq 0.25$ cold dark matter model or mixed dark matter model with $20 \%$ of cold dark matter. The heavy stable uniton is a good candidate of the cold dark matter component. We conclude that the uniton can evade all the currently available experimental constraints. We therefore propose that the uniton is a good dark matter candidate.

The consistency of perturbative string unification with low energy data seems to require the existence of additional intermediate matter thresholds [17. Remarkably, the same states that are required for the string scale unification, solve at the same time the dark matter problem. As there exist many possible scenarios for the scales of the additional matter states [1], the string scale unification constraints can be compatible with the constraints on the uniton dark matter [3]. In the free fermionic standardlike models the additional matter states are obtained from sectors that correspond to "Wilson lines" in orbifold models. It is well known that the "Wilson line" breaking in superstring models results in physical states with fractional electric charge [18. Due to electric charge conservation, fractionally charged states are stable. As there exist strong constraints on their masses and abundance, fractionally charged states cannot constitute the dark matter. Such states must be diluted away or extremely massive. Remarkably, however, the same "Wilson line" breaking mechanism, that produces matter with fractional electric charge, is also responsible for the existence of states which carry the "standard" charges under the Standard Model gauge group but carry fractional charges under the $U(1)_{Z^{\prime}}$ symmetry. In the free fermionic standard-like models the three light generations are obtained from the three 16 representations of $S O(10)$. Consequently, due to the $U(1)_{Z^{\prime}}$ charge conservation, the additional matter states cannot decay into the standard model states. This may be the case even after $U(1)_{Z^{\prime}}$ symmetry breaking, in which case a local discrete symmetry is left unbroken. 
It is very encouraging, in our opinion, that the stability of the uniton is associated with a gauge symmetry or a local discrete symmetry. As global symmetries are in general expected to be violated by quantum gravity effects, this fact is an important advantage over some other dark matter candidates. Due to the general applicability of the "Wilson line" breaking mechanism in superstring models, the uniton may in fact be generic to string models that aim at obtaining the standard model gauge group directly at the string scale. It will be of further interest to study additional cosmological and phenomenological implications that this type of matter might have. Such work is in progress.

We thank G. Bodwin, R. Field, D. Kennedy, P. Ramond, P. Sikivie and C. Thorn for discussions. This work was supported in part by DOE Grant No. DEFG-0586ER40272 and by KOSEF.

\section{References}

[1] K.R. Dienes and A.E. Faraggi, Phys. Rev. Lett. 75, 2646 (1995); Nucl. Phys. B457, 409 (1995).

[2] L.M. Krauss and F. Wilzcek, Phys. Rev. Lett. 62, 1221 (1989).

[3] S. Chang, C. Corianò and A.E. Faraggi, Nucl. Phys. B477, 65 (1996).

[4] P. Ginsparg, Phys. Lett. B197, 139 (1987); V.S. Kaplunovsky, Nucl. Phys. B307, 145 (1988); Erratum: ibid., B382, 436 (1992).

[5] M.K. Gaillard and R. Xiu, Phys. Lett. B296, 71 (1992); J. Lopez, D. Nanopoulos, and K. Yuan, Nucl. Phys. B399, 654 (1993); S.P. Martin and P. Ramond, Phys. Rev. D51, 6515 (1995).

[6] A.E. Faraggi, Phys. Lett. B302, 202 (1993).

[7] H. Kawai, D.C. Lewellen, and S.-H.H. Tye, Nucl. Phys. B288, 1 (1987); I. Antoniadis, C. Bachas, and C. Kounnas, Nucl. Phys. B289, 87 (1987). 
[8] I. Antoniadis et al, Phys. Lett. B231, 65 (1989); A.E. Faraggi, Nucl. Phys. B387, 239 (1992).

[9] I. Antoniadis, G.K. Leontaris and J. Rizos, Phys. Lett. B245, 161 (1990).

[10] A.E. Faraggi, Phys. Lett. B278, 131 (1992).

[11] A.E. Faraggi, Nucl. Phys. B428, 111 (1994).

[12] M. Luty and R. Sundrum, Phys. Rev. D52, 1627 (1995)

[13] Particle Data Group, L. Montanet et al, Phys. Rev. D50, 1173 (1994).

[14] E. W. Kolb and M. S. Turner, The Early Universe (Addison-Wesley, New York, 1990).

[15] G. D. Starkman, A. Gould, R. Esmailzadeh and S. Dimopoulos, Phys. Rev. D41, 3594 (1990).

[16] A. Gould, G. Draine, R. Romani and S. Nussinov, Phys. Lett. B238, 337 (1990).

[17] For an alternative proposal based on strongly interacting string see E. Witten, hep-th/9602070.

[18] E. Witten and X.G. Wen, Nucl. Phys. B261, 651 (1985). 